H.Н.Мазитова ${ }^{1}$, A.A.Савельев ${ }^{2}$

\title{
Цитокиновый статус рабочих как отражение восприимчивости органов дыхания к воздействию кварцсодержащих промышленных аэрозолей
}

1 - Кафедра гигиены, медицины труда ГОУ ВПО "Казанский государственный медицинский университет Росздрава": 420012, Республика Татарстан, Казань, ул. Бутлерова, 49;

2 - Кафедра моделирования экологических систем Казанского (Приволжского) Федерального университета: 420008, Республика Татарстан, Казань, ул. Кремлевская, 18

N.N.Mazitova, A.A.Savelyev Cytokine status in workers as marker of respiratory
responsibility to exposure to quartz-containing industrial
aerosols

Summary

Eighty-eight men were involved in the study and divided into groups as follows: the $1^{\text {st }}$ control group (patients with COPD, $n=8$ ), the $2^{\text {nd }}$ control group (healthy foundry workers, $n=11)$, patients with occupational bronchitis $(n=33)$, and patients with occupational COPD $(n=36)$. All workers had occupational exposure to the silica dust at the foundry industry. Levels of interleukin (IL)- $1 \beta$, IL-6, IL-8, tumor necrosis factor, interferon- $\gamma, \alpha$-antitrypsin and lung function were analyzed. A high variability of inflammatory response was detected in occupational COPD. The most significant predictor of occupational COPD progression was IL- $1 \beta$. Dose-effect relationship between silica dust exposure and development of occupational COPD was confirmed. The results of the study showed the equal importance of smoking and exposure to silica dust for development of occupational COPD.

Key words: chronic obstructive pulmonary disease, cytokines, silica dust, foundry workers.

\section{Резюме}

88 мужчин были распределены в следующие группы: 1-я контрольная группа (8 пациентов с хронической обструктивной болезнью легких (ХОБЛ), курильщики), 2-я контрольная группа (11 здоровых рабочих-металлургов) и 2 группы больных: хроническим профессиональным необструктивным бронхитом $(n=33)$ и ХОБЛ профессиональной этиологии (ХОБЛ ПЭ) $(n=36)$. Все рабочие имели профессиональный контакт с кварцсодержащими промышленными аэрозолями (ПА) на металлургическом производстве. Анализировались уровни интерлейкинов: IL-1 $\beta$, IL-6, IL-8, фактора некроза опухоли, интерферона- $\gamma, \alpha_{1}$-антитрипсина, показатели вентиляционной функции легких. Обнаружена высокая вариабельность воспалительного ответа при ХОБЛ ПЭ, выявлен наиболее значимый предиктор ее прогрессирования - IL-1 $\beta$, подтверждена зависимость "доза-эффект" между воздействием кварцсодержащих ПА и развитием ХОБЛ ПЭ. Полученные результаты показали примерно одинаковую значимость эффектов курения и воздействия кварцевой пыли на развитие профессионального бронхита.

Ключевые слова: хроническая обструктивная болезнь легких, цитокины, кварцевая пыль, работающие на металлургическом производстве.

Ведущим механизмом патогенеза хронической обструктивной болезни легких (ХОБЛ) является хронический воспалительный ответ легких на вдыхание токсических частиц и газов, содержащихся в табачном дыме, загрязненном атмосферном воздухе и / или воздухе производственных помещений [1]. Профессиональные факторы являются важными факторами риска ХОБЛ, а кварцевая пыль - одним из наиболее важных профессиональных факторов, оказывающих влияние на респираторную систему [2].

Интенсивность воспалительного ответа при ХОБЛ зависит, во многом, от местной и системной продукции провоспалительных цитокинов - интерлейкинов (IL): IL- $1 \beta$, IL-6, IL-8 и фактора некроза опухолей (TNF) [1]. Воспалительный ответ наблюдается не только во время контакта со вредными частицами и газами, но и после него [3]. Данный феномен свидетельствует о возможном нарушении регуляции воспалительного ответа в легких у восприимчивых лиц. Однако факторы, определяющие восприимчивость, недостаточно изучены [4].

Известно, что воспалительный ответ при профессиональных респираторных заболеваниях характеризуется, как и при ХОБЛ, значительной концентрацией макрофагов в тканях и продукцией ими провоспалительных цитокинов [5]. Описано несколько возможных механизмов, с помощью которых частицы кварцевой пыли могут инициировать активацию макрофагов и клеточное воспаление, приводящее к развитию ХОБЛ. К ним относится высокая цитотоксичность, приводящая к образованию активных форм кислорода и секреции провоспалительных факторов, в т. ч. цитокинов [2]. Известно также о зависимости "доза-эффект" между величиной пылевой нагрузки и развитием клиники ХОБЛ у работающих $[2,6]$. 
Однако до сегодняшнего времени отсутствуют надежные биомаркеры ранней диагностики ХОБЛ профессиональной этиологии, в то время как таковые хорошо известны для оценки прогноза легочной функции, обострений и смертности при ХОБЛ курильщика $[8,9]$. В связи с этим является актуальной задача поиска наиболее чувствительного, информативного и удобного в исследовании биомаркера воспаления при ХОБЛ, вызванной профессиональными факторами, поскольку диагностика данного вида профессиональной респираторной патологии в настоящее время осуществляется только на основании эпидемиологических данных [10, 11], что не соответствует критериям доказательной медицины.

С 2002 г. общепринятым в мире стал термин "occupational COРD" (ХОБЛ профессиональной этиологии, далее - ХОБЛ ПЭ) [7], в отличие от термина "occupational bronchitis" (профессиональный бронхит), обозначающего вариант хронического простого (необструктивного) бронхита, развившегося под действием профессиональных этиологических факторов. Разница между этими нозологическими формами представляется очевидной вследствие несходства в течении и исходах, а также подходах к терапии этих заболеваний. Однако в практике отечественной профпатологической службы до сих пор употребляется понятие "профессиональный бронхит", объединяющее в себе обе указанные нозологии.

Целью исследования было изучение особенностей продукции провоспалительных цитокинов у больных с 2 типами течения профессиональных бронхитов: хроническим профессиональным (необструктивным) бронхитом и ХОБЛ профессиональной этиологии), для выявления зависимости между уровнем цитокинов и вариантом развития профессиональной патологии бронхиального дерева.

\section{Материалы и методы}

Проведено поперечное исследование, критериями включения в которое явились добровольное информированное согласие на участие в исследовании, профессиональный стаж в контакте с кварцсодержащими промышленными аэрозолями (ПА) на металлургическом производстве $\geq 10$ лет и наличие кашлевого анамнеза, соответствующего определению хронического бронхита по критериям ВОЗ. Кроме того, в данное исследование были включены 8 больных непрофессиональной ХОБЛ, не имевших контакта с ПА в течение жизни, составившие 1-ю контрольную группу.

Всего были обследованы 88 мужчин, средний возраст которых составил 51,4 лет (95\%-ный доверительный интервал (ДИ) - 50,2-52,7), средний профессиональный стаж - 21,6 лет (95\%-ный ДИ 19,6-23,5). Из них некурящие составили 23,86 \%, курящие - 36,36 \%, экс-курильщики - 39,77 \%; индекс курильщика (количество пачко-лет) составил в среднем 21,3 (95\%-ный ДИ - 17,7-25,6).

На момент обследования все пациенты находились в стадии ремиссии, в т. ч. 33 больных хроничес- ким профессиональным необструктивным бронхитом (далее - ХПБ) и 36 больных ХОБЛ ПЭ. У 11 пациентов отсутствовали признаки респираторной патологии при клиническом и функциональном обследовании: они составили 2-ю контрольную группу. Сведения об уровнях ПА были получены из карт аттестации рабочих мест и протоколов исследования воздуха рабочей зоны, предоставленных работодателем. Средняя кратность превышения среднесуточной предельно допустимой концентрации (ПДКсс) кварцсодержащей пыли составила 7,5 (95\%-ный ДИ - 5,9-9,0), при средней кратности превышения контрольной пылевой нагрузки (КПН) равной 5,6 $(95 \%$-ный ДИ - 3,8-7,5) и средней кратности превышения ПДК раздражающих веществ с учетом суммации равной 0,73 (95\%-ный ДИ - 0,32-1,14).

Забор венозной крови и исследование вентиляционной функции легких всем рабочим проводили в конце рабочей смены. Определение концентрации IL-1 $\beta$, IL-6, IL-8, TNF, интерферона- $\gamma$ (IFN- $\gamma$ ) в сыворотке крови проводили твердофазным иммуноферментным методом (ИФА) с использованием тестсистем ООО "Цитокин" (Санкт-Петербург). Уровень $\alpha_{1}$-антитрипсина ( $\alpha_{1}$-АТ) определяли иммунотурбидиметрическим методом с помощью реактивов фирмы Spinreact (Испания). Исследование вентиляционной функции легких проводилось с помощью спирометра Spirolab III (MIR, Италия).

Для проверки истинности гипотезы о наличии связи между высоким уровнем цитокинов и развитием профессиональной респираторной патологии по типу ХОБЛ ПЭ нами была построена логистическая модель регрессионного анализа, имеющая следующий вид:

$$
\begin{aligned}
& \operatorname{logit}(Y)=a_{0}+a_{1} X_{1}+\ldots+a_{\mathrm{n}} X_{\mathrm{n}} \\
& \operatorname{logit}(Y)=\ln \left(\frac{P(Y)}{1-P(Y)}\right),
\end{aligned}
$$

где $a_{0}+a_{1} X_{1}+a_{\mathrm{n}} X_{\mathrm{n}}-$ линейные предикторы (исследуемые цитокины);

$P(Y)$ - вероятность развития ХОБЛ ПЭ.

Прогнозная вероятность, таким образом, имела следующий вид:

$$
P(Y)=\frac{1}{1+\mathrm{e}^{-\left(a_{0}+a_{1} X_{1}+\ldots+a_{n} X_{n}\right)}}
$$

Для статистической обработки данных использовались стандартный пакет программ Statistica for Windows 7.0 и статистическая система $R$ ( $R$ Development Core Team, 2008) [12]. Проведена проверка законов распределения исследуемых переменных на нормальность по критериям Колмогорова-Смирнова и Лиллиефорса: по всем зависимым переменным полученные значения укладывались в признаки нормального распределения. Проверка когорты обследованных рабочих на однородность показала отсутствие значимых $(p<0,05)$ различий по таким признакам, как возраст, стаж, индекс курильщика. Для сравнения различий средних использовался независимый $\mathrm{t}$-тест для групп. Для построения моделей применял- 
ся метод множественной регрессии: логистической для категориальных, обычной - для скалярных зависимых переменных.

\section{Результаты и обсуждение}

У обследованных рабочих, имевших контакт с кварцсодержащими промышленными аэрозолями, наблюдалась высокая вариабельность выраженности воспалительного ответа, равная 2-3 порядкам величин, в особенности для IL-6 и IFN- $\gamma$ (табл. 1), в отличие от больных ХОБЛ курильщика, у которых диапазон колебаний уровня цитокинов укладывался в пределы 2 норм показателей. Были обнаружены статистически значимые зависимости между уровнями всех исследованных провоспалительных цитокинов с наибольшими значениями коэффициентов парной корреляции Пирсона у IL-6 и TNF $(r=0,7080, p<0,0001)$, IL-1 и TNF $(r=0,6174$; $p<0,0001)$, IL-1 и IL-6 $(r=0,6025 ; p<0,0001)$.

Уровень провоспалительных цитокинов был повышен, хотя и в разной степени, у всех работающих в контакте с кварцсодержащими промышленными аэрозолями (табл. 2). При этом максимальные концентрации наблюдались у больных ХОБЛ ПЭ, на 2-м месте по величине цитокинового ответа оказались больные ХПБ, на 3-м - здоровые рабочие (2-я контрольная группа), минимальные концентрации цитокинов были обнаружены в крови у больных ХОБЛ курильщика (1-я контрольная группа). Различия между исследуемыми группами были наиболее выраженными для IL- $1 \beta$, IL-8 и IFN- $\gamma$.

В качестве зависимой переменной была принята принадлежность рабочего к одной из следующих групп: здоровые рабочие; больные ХПБ; больные ХОБЛ ПЭ. Поскольку различия между здоровыми рабочими и больными ХПБ оказались статистически незначимыми, указанные группы были объе-

Таблица 1

Вариабельность цитокинового ответа у обследованных рабочих (пг / мл)

\begin{tabular}{|l|c|c|c|c|} 
& $M(S D)$ & Медиана & Минимум & Максимум \\
\hline IL-1 $\beta$ & $56,6(44,0)$ & 44,0 & 2,1 & 226,7 \\
IL-6 & $241,9(321,2)$ & 117,6 & 19,8 & 1780,8 \\
IL-8 & $86,9(73,5)$ & 63,8 & 5,8 & 496,4 \\
\hline TNF & $86,9(37,2)$ & 83,8 & 17,5 & 268,3 \\
\hline IFN- $\gamma$ & $174,6(173,6)$ & 131,7 & 24,4 & 1082,8 \\
\hline$\alpha_{1}$-AT & $3,0(0,7)$ & 3,2 & 1,5 & 4,9
\end{tabular}

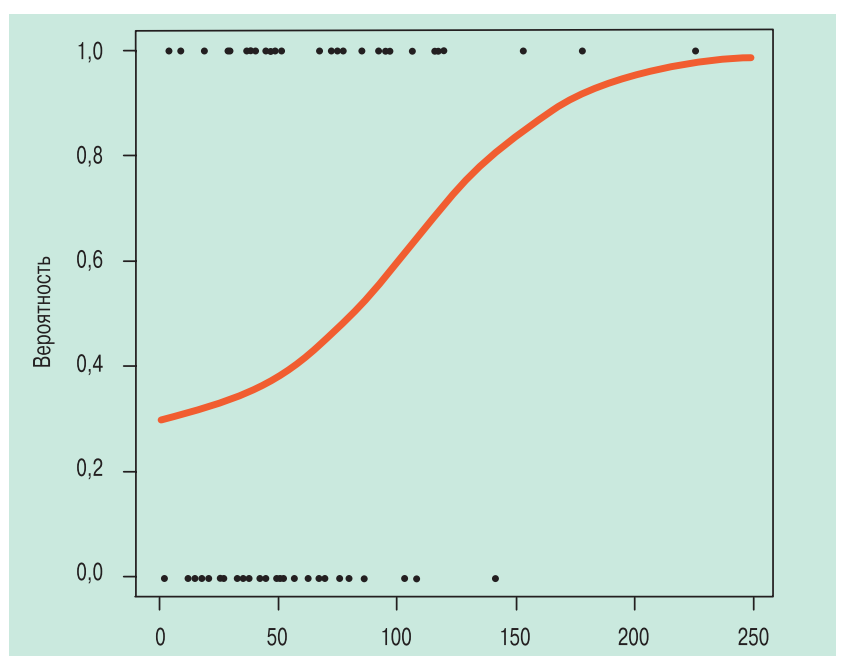

Рисунок. Вероятность развития ХОБЛ ПЭ в зависимости от уровня IL- $1 \beta$

динены для анализа. Использовалась процедура последовательного включения переменных в модель, поэтому из всех коррелированных переменных в модель включалась только наиболее значимая. Так, из всего перечня исследовавшихся цитокинов в итоговую модель был включен 1 наиболее значимый предиктор - IL-1 $\beta$ ( $p<0,05)$. При этом наблюдалась более слабая, но также значимая $(p<0,05)$ статистическая зависимость для IL-8 и TNF. В связи с наличием высокой степени корреляции названных цитокинов $(r>0,6)$ модель зависимости могла быть описана одной, наиболее сильно влияющей, переменной - IL-1 $\beta$ (рисунок), с ростом уровня которой вероятность развития ХОБЛ ПЭ возрастает.

В нелинейной аддитивной модели, описывающей зависимость выраженности воспалительного ответа цитокинов и показателей вентиляционной функции легких, статистически значимыми предикторами для значения ОФВ / ФЖЕЛ оказались уровень TNF и IFN- $\alpha$ ( $p$-value $=0,02$ и 0,03 соответственно), для ОФВ 1 - только IFN- $\gamma(p$-value $=0,0007)$. По другим показателям значимых предикторов выявлено не было.

Гипотеза о наличии статистической связи между факторами профессионального риска и выраженностью респираторной патологии требовала уточнения. Для этого была построена логистическая модель регрессионного анализа с предикторами, описывающими условия труда (профессиональный стаж, класс условий труда по Р 2.2.2006-05, крат-

Таблица 2

Уровни провоспалительных цитокинов в группах (M (SD), пг / мл)

\begin{tabular}{|l|c|c|c|c|} 
& 1-я контрольная группа, $n=8$ & 2 -я контрольная группа, $n=11$ & Больные XПБ, $n=33$ & Больные ХОБл пЭ, $n=36$ \\
\hline IL-1 $\beta$ & $18,6(12,6)$ & $40,4(37,1)^{*}$ & $50,1(27,4)$ & $75,9(53,5)^{*}$ \\
\hline IL-6 & $124,6(87,7)$ & $124,3(59,1)$ & $201,5(226,1)$ & $340,9(433,6)$ \\
\hline IL-8 & $40,8(21,9)$ & $54,2(39,0)^{*}$ & $81,1(61,1)$ & $112,4(89,2)^{*}$ \\
\hline TNF & $89,7(14,7)$ & $80,9(13,9)$ & $72,9(24,9)$ & $101,0(48,7)$ \\
\hline IFN- $\gamma$ & $65,7(36,2)$ & $94,7(39,9)^{*}$ & $140,9(62,3)^{*}$ & $254,1(241,6)^{*}$ \\
\hline
\end{tabular}

Примечание: 1-я контрольная группа - больные ХОБЛ курильщика; 2-я контрольная группа - здоровые рабочие; * - значение p-value < 0,05 (независимый t-тест) между отмеченными значениями. 
Таблица 3

Детерминанты воспалительного ответа

\begin{tabular}{|c|c|c|c|c|c|c|}
\hline & IL-1 $\beta$ & IL-6 & IL-8 & TNF & IFN- $\gamma$ & $\alpha_{1}$-AT \\
\hline Класс условий труда & & & 0,01 & & & \\
\hline Профессиональный стаж & & & & & & 0,0006 \\
\hline Удельный вес $\mathrm{SiO}_{2}$ в ПА & 0,05 & & & & & \\
\hline Контакт с ПА за смену, \% & & & & & 0,002 & \\
\hline Кратность превышения ПДКсс & & & 0,01 & & & \\
\hline Кратность превышения КПН & 0,006 & 0,05 & & & & \\
\hline $\begin{array}{l}\text { Средняя кратность превышения ПДК } \\
\text { раздражающих веществ }\end{array}$ & & & & & & 0,05 \\
\hline
\end{tabular}

Примечание: в ячейках приведен уровень значимости; указаны только значения p-value $<0,05$.

ность превышения среднесменной ПДК, кратность превышения КПН, вид ПА, процент контакта с ним в течение смены, суммарную кратность превышения ПДК раздражающих веществ, наличие перегрева и физического напряжения). В качестве зависимых переменных $Y$ были приняты вероятность развития ХОБЛ ПЭ и вероятность воспалительного отклика цитокинов на изменение условий труда. При этом за наличие отклика нами принимались случаи, когда концентрация цитокина превышала значение его медианы.

В модели вероятности развития ХОБЛ ПЭ значимыми $(p<0,05)$ признаками явились кратность превышения КПН ( $p=0,03 ; z$-value $=2,167)$ и профессиональный стаж ( $p=0,02 ; z$-value $=2,228)$. Аналогичная логистическая модель, описывающая вероятность ответа цитокинов на изменение условий труда, показала наличие влияния различных переменных (табл. 3), при этом статистически наиболее значимыми предикторами явились также кратность превышения КПН и профессиональный стаж. Уровень $\alpha_{1}$-АТ также сильно зависел $(p=0,0006)$ от длительности профессионального стажа в контакте с кварцсодержащими ПА.

Параллельно была построена логистическая модель, описывающая зависимость развития профессиональной респираторной патологии от курения, в качестве предикторов которой были приняты статус курения (курильщик, экс-курильщик, некурящий), длительность курения и индекс курильщика (количество пачко-лет). Статистически значимым предиктором в данной модели оказался индекс курильщика $(p$-value $=0,02 ; z$-value $=2,13)$. В модели, описывающей влияние курения на выраженность воспалительного ответа цитокинов и $\alpha_{1}$-AТ, значимые предикторы были выявлены только для ТNF (индекс курильщика, $p<0,012$ ). Для IL- $\beta$ зависимость от факта курения имелась, но была слабозначимой $(p<0,08)$.

\section{Заключение}

В исследовании обнаружена высокая вариабельность воспалительного ответа у работающих в контакте с кварцсодержащими промышленными аэрозолями, отсутствовавшая у больных ХОБЛ курильщика в стадии ремиссии. Учитывая высокую скорость синтеза цитокинов в организме, можно полагать, что забор крови непосредственно на рабочем месте и в конце рабочего дня позволяет оценить индуцированную ПА выработку этих факторов воспаления. Обнаружены статистически достоверные корреляции между всеми исследованными провоспалительными цитокинами: т. о., еще раз подтверждено известное положение о некоторой избыточности функций в семействе цитокинов, когда каждая их них потенциально регулируется более чем 1 цитокином [13].

Уровень провоспалительных цитокинов был повышен, хотя и в разной степени, у всех работающих, что соответствует существующим представлениям о патогенезе процессов, происходящих при действии кварцсодержащих ПА [14]. При этом максимальные уровни цитокинов были обнаружены у больных ХОБЛ ПЭ, а минимальные - у больных ХОБЛ курильщика в стадии ремиссии. Полученные результаты заставляют задуматься о целесообразности коррекции единого подхода к экспертизе трудоспособности при профессиональных бронхитах. Высокая интенсивность воспалительного процесса, развивающегося в легких у больных ХОБЛ ПЭ при условии продолжения контакта с ПА, неизбежно приводит к быстрому прогрессированию болезни и развитию стойкой утраты трудоспособности, как это уже было нами показано ранее по результатам длительного проспективного наблюдения [15].

Логистическая модель регрессионного анализа позволила выявить наиболее значимый предиктор развития ХОБЛ ПЭ - IL- $\beta$, рост концентрации которого приводит к увеличению вероятности развития ХОБЛ ПЭ в модели. Кроме того, при анализе обнаружено отсутствие статистически значимых различий в характере воспалительного ответа между здоровыми рабочими и больными ХПБ, что свидетельствует о важных различиях в типе воспалительного ответа при ХПБ и ХОБЛ ПЭ. В логистической модели вероятности развития ХОБЛ ПЭ от предикторов, описывающих условия труда, значимыми переменными явились кратность превышения КПН $(p$-value $=0,03 ; z$-value $=2,167)$ и профессиональный стаж $(p$-value $=0,02 ; z$-value $=2,228)$. Аналогичная логистическая модель, описывающая вероятность отклика цитокинов на изменение условий труда, подтвердила значимость тех же детерминант для выраженности цитокинового воспалительного ответа. 
Таким образом, подтверждается наличие зависимости "доза-эффект" между воздействием ПА и развитием ХОБЛ ПЭ. Величина значимости индекса курильщика $(p$-value $=0,02 ; z$-value $=2,13)$ позволила определить вероятность развития респираторной патологии у работающих, обусловленную курением. Таким образом, значимость эффектов курения и воздействия кварцсодержащих ПА в развитии ХОБЛ ПЭ, полученная в настоящем исследовании, примерно одинакова.

Сильная степень зависимости уровня $\alpha_{1}$-АТ $(p=0,0006)$ от длительности профессионального стажа в контакте с кварцсодержащими ПА согласуется с ролью этого тканевого ингибитора протеаз, одного из острофазных белков, концентрация которого повышается при воспалительных процессах.

Необходимо дальнейшее изучение возможной роли IL-1 $\beta$ как биомаркера воспалительного ответа при ХОБЛ, обусловленной воздействием ПА, поскольку данным исследованием сделан только 1-й шаг - очерчен круг потенциальных предикторов. Для построения надежной прогностической модели необходимо увеличение объема выборки и дальнейшие исследования.

\section{Литература}

1. Reid P.T., Sallenave J.M. Cytokines in the pathogenesis of chronic obstructive pulmonary disease. Curr. Pharm. Des. 2003; 9: 25-38.

2. Hnizdo E., Vallyathan $V$. Chronic obstructive pulmonary disease due to occupational exposure to silica dust: a review of epidemiological and pathological evidence. Occup. Environ. Med. 2003; 60: 237-243.

3. Willemse B.W., ten Hacken N.H., Rutgers B. et al. Effect of 1 -year smoking cessation on airway inflammation in COPD and asymptomatic smokers. Eur. Respir. J. 2005; 26: 835-845.

4. MacNee $W$. Pathogenesis of chronic obstructive pulmonary disease. Clin. Chest Med. 2007; 28: 479-513.

5. Adelroth E., Hedlund U., Blomberg A. et al. Airway inflammation in iron ore miners exposed to dust and diesel exhaust. Eur. Respir. J. 2006; 27: 714-719.
6. Kuempel E.D., Attfield M.D., Vallyathan V. et al. Pulmonary inflammation and crystalline silica in respirable coal mine dust: Dose response. J. Biosci. 2003; 28 (1): 61-69.

7. International Labour Conference. Recommendation 194. Recommendation concerning the list of occupational diseases and the recording and notification of occupational accidents and diseases, adopted by the Conference at its ninetieth session, Geneva, 20 June 2002. Geneva; 2002.

8. Pinto-Plata V., Toso J., Lee K. et al. Profiling serum biomarkers in patients with COPD: associations with clinical parameters. Thorax 2007; 62: 595-601.

9. Hurst J.R., Donaldson G.C., Perera W.R. et al. Use of plasma biomarkers at exacerbation of chronic obstructive pulmonary disease. Am. J. Respir. Crit. Care Med. 2006; 174: 867-874.

10. Boschetto P., Quintavalle S., Miotto D. et al. Chronic obstructive pulmonary disease (COPD) and occupational exposures. J. Occup. Med. Toxicol. 2006; 7 (1): 11.

11. Ameille J., Dalphin J.C., Descatha A. et al. La bronchopneumopathie chronique obstructive professionnelle: une maladie meconnue. Rev. Mal. Respir. 2006; 23: 13S119-13S130.

12. R: A language and environment for statistical computing. Vienna: R Foundation for Statistical Computing. Vienna, Austria; 2008.

13. Гердт Л.В., Черемисина О.В., Стахеева М.Н. и др. Цитокиновый профиль у больных простым хроническим бронхитом с диспластическими изменениями бронхиального эпителия. Сиб. онкол. журн. 2004; 4 (120): 48-52.

14. Величковский Б.Т. Экологическая пульмонология (роль свободнорадикальных процессов). Екатеринбург: Издание ЕМНЦ ПОЗРПП Минздрава России; 2003.

15. Амиров Н.Х., Мазитова Н.Н., Берхеева З.М. и др. Результаты длительного проспективного наблюдения больных с хроническими профессиональными бронхитами. Казан. мед. журн. 2009; 90 (4): 513-518.

\section{Информация об авторах}

Мазитова Наиля Наилевна - к. м. н., докторант кафедры гигиены, медицины труда ГОУ ВПО "Казанский государственный медицинский университет Росздрава"; тел. / Факс: (843)-238-29-58, e-mail: mazitova_ nailya@mail.ru

Савельев Анатолий Александрович - д. б. н., проф. кафедры моделирования экологических систем Казанского (Приволжского) Федерального университета; тел.: (843) 264-47-44; e-mail: Anatoly.Saveliev @ksu.ru

Поступила 09.02.10 () Мазитова Н.Н., Савельев А.А., 2010 УДК 616.2-057-092 